\title{
(GIGA)bYte
}

\section{Genome assembly and annotation of the tambaqui (Colossoma macropomum): an emblematic fish of the Amazon River Basin}

\author{
Alexandre Wagner Silva Hilsdorf ${ }^{1, *, \dagger}$, Marcela Uliano-Silva ${ }^{2, \dagger}$, \\ Luiz Lehmann Coutinho ${ }^{3}$, Horácio Montenegro ${ }^{3}$, \\ Vera Maria Fonseca Almeida-Val ${ }^{4}$ and Danillo Pinhal ${ }^{5, *}$
}

1 Integrated Center of Biotechnology, University of Mogi das Cruzes, P.O. Box 411, Mogi das Cruzes, SP 08780-911, Brazil

2 Wellcome Sanger Institute, Saffron Walden, Hinxton, Cambridgeshire CB10 1SA, UK

3 Animal Science Department, University of São Paulo (USP)/Luiz de Queiroz College of Agriculture (ESALQ), Piracicaba, SP 13418-900, Brazil

4 Brazilian National Institute for Research of the Amazon, Laboratory of Ecophysiology and Molecular Evolution, Manaus, AM 69067-375, Brazil

5 Department of Chemical and Biological Sciences, Institute of Biosciences of Botucatu, São Paulo State University (UNESP), Botucatu, SP 18618-689, Brazil

\section{ABSTRACT}

Colossoma macropomum, known as "tambaqui", is the largest Characiformes fish in the Amazon River Basin and a leading species in Brazilian aquaculture and fisheries. Good quality meat and excellent adaptability to culture systems are some of its remarkable farming features. To support studies into the genetics and genomics of the tambaqui, we have produced the first high-quality genome for the species. We combined Illumina and PacBio sequencing technologies to generate a reference genome, assembled with $39 \times$ coverage of long reads and polished to a consensus quality value (QV) of 36 with $130 \times$ coverage of short reads. The genome was assembled into 1269 scaffolds (a total of 1,221,847,006 bases), with a scaffold N50 size of $40 \mathrm{Mb}$, where $93 \%$ of all

Submitted: $\quad 20$ July 2021

Accepted: $\quad 22$ September 2021

Published: $\quad 27$ September 2021

* Corresponding authors. E-mail: wagner@umc.br;

danillo.pinhal@unesp.br

$\dagger$ Contributed equally.

Published by GigaScience Press.

Preprint submitted at

https://www.biorxiv.org/content/10. 1101/2021.09.08.459456v1

This is an Open Access article distributed under the terms of the Creative Commons Attribution License (http://creativecommons.org/ licenses/by/4.0/), which permits unrestricted reuse, distribution, and reproduction in any medium, provided the original work is properly cited.

Gigabyte, 2021, 1-14 assembled bases were placed in the largest 54 scaffolds corresponding to the diploid karyotype of the tambaqui. Furthermore, the NCBI Annotation Pipeline annotated genes, pseudogenes, and non-coding transcripts using the RefSeq database as evidence, guaranteeing a high-quality annotation. A Genome Data Viewer for the tambaqui was produced, which will benefit groups interested in exploring the unique genomic features of the species. The availability of a highly accurate genome assembly for tambaqui provides the foundation for the discovery of novel ecological and evolutionary insights, and is a helpful resource for aquaculture.

Subjects Genetics and Genomics, Evolutionary Biology, Marine Biology

\section{INTRODUCTION}

The Amazon Basin harbors enormous freshwater ichthyo diversity throughout its rivers and tributaries, with 2406 validated freshwater native fish species from 232,936 georeferenced records [1]. Colossoma macropomum (NCBI:txid42526, fishbase ID:263) is the largest Characiformes representative found across the Amazon River and its tributaries, with individuals reaching 1 meter in length and $30 \mathrm{~kg}$ in weight (Figure 1) [2]. This species is known by different common names, such as "tambaqui" in Brazil and "cachama Negra" in 


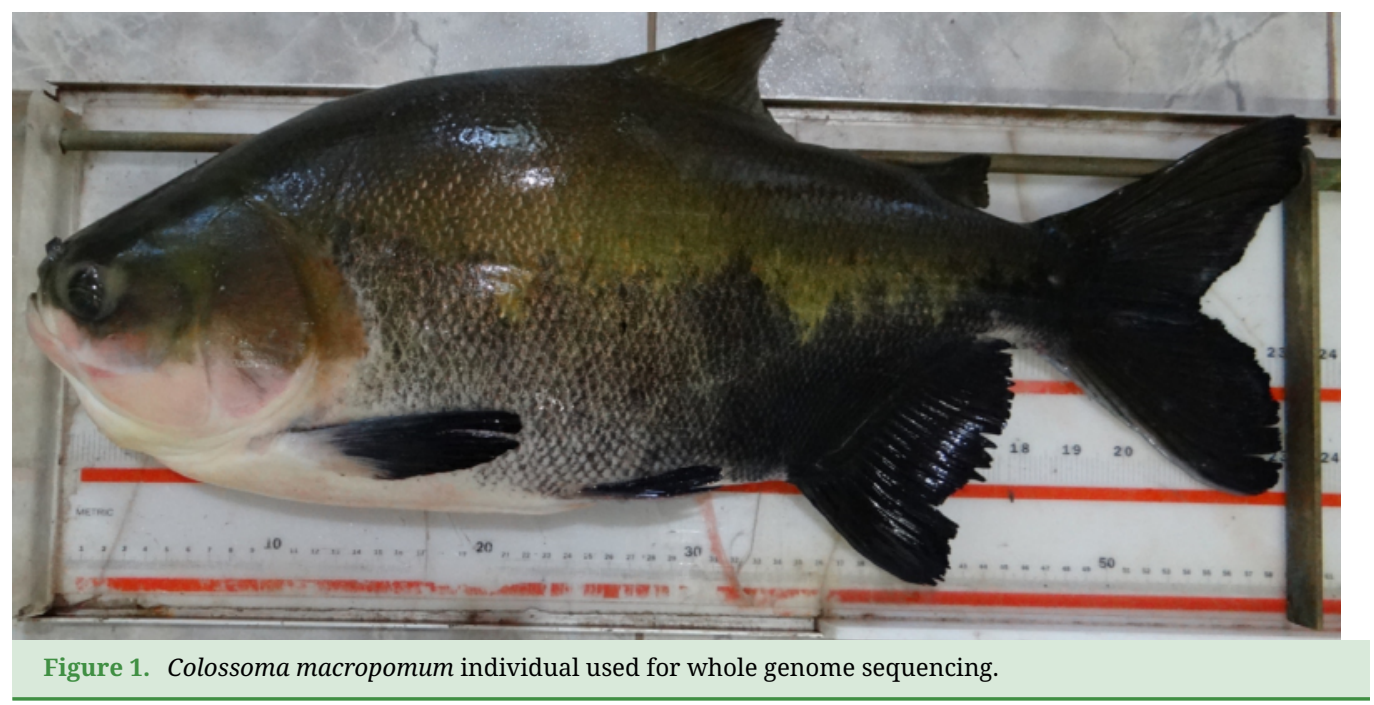

Colombia. Tambaquis are omnivore/frugivore benthopelagic fish, and they have an essential ecological role as seed dispersers [3]. They are potamodromous fish, with upstream migration and reproduction taking place in the white waters along woody shores between November and February [4]. The tambaqui is an important food and income source for Amazonian fishing communities; it is the most frequently farmed native fish species in Brazil, with a production of 101,079 metric tons in 2019 [5, 6].

The ecological and economic importance of the tambaqui means it is a comparatively well-studied species. Research to date has focused on its biological adaptations to the Amazon River waters, and on the genetics of production traits to assist selective breeding programs. Transcriptomic characterization of tambaqui exposed to (i) distinct climate change scenarios, and (ii) during gonadal differentiation, has provided helpful resources for understanding the molecular mechanisms underlying both adaptation to a future new climate and the process of sex determination [7-9]. Other molecular mechanisms related to enzymatic capacity for long-chain polyunsaturated fatty acid biosynthesis have also been confirmed by the functional characterization of core genes in these processes [10,11]. The first steps for deciphering the structure and functional dynamics of the tambaqui genome have already been taken, with large-scale single nucleotide polymorphism (SNP) discovery allowing a high-density genetic linkage map of the species to be built [12], along with preliminary microRNA identification and characterization [13]. Equally pertinent are the new findings in morphology: specimens lacking intramuscular bones were identified in a fish farm in Brazil; however, the genetic and molecular mechanisms underlying the expression of such desirable phenotypes for the fish market remain unknown [14, 15].

Considering the great need for increased genetic resources for the tambaqui to assist fishery management and aquaculture [16], here we present the first high-quality reference genome for $C$. macropomum. This complete set of DNA provides a valuable resource for the study of evolutionary and functional genomics in bony fishes, providing a window of opportunity to reveal singularities of the tambaqui genome, as well as to help develop molecular techniques to improve selective breeding programs. 


\begin{tabular}{|c|c|c|c|c|c|}
\hline Genes & COI & TROP & fkh & RAG2 & sina \\
\hline \multicolumn{6}{|l|}{ Species } \\
\hline Colossoma macropomum & HQ420845.1 & HQ420888.1 & AY817328.1 & AY804061.1 & AY790059.1 \\
\hline Piaractus brachypomus & HQ420838.1 & HQ420883.1 & AY817392.1 & AY804112.1 & AY790125.1 \\
\hline Piaractus mesopotamicus & HQ420837.1 & HQ420878.1 & AY817398.1 & AY804118.1 & AY790131.1 \\
\hline
\end{tabular}

\begin{tabular}{lcccc|}
\hline \multicolumn{5}{l}{ Table 2. Final statistics for the genome assembly of Colossoma macropomum. } \\
\hline Contig length (bp) & Scaffold length (bp) & Number of Contigs & Number of Scaffolds \\
Total & $1,221,809,066$ & $1,221,847,006$ & 1687 & 1269 \\
Max & $26,165,397$ & $63,817,184$ & - & - \\
N50 & $5,645,235$ & $40,163,545$ & 54 & 14 \\
N90 & 655,952 & $2,856,822$ & 300 & 33 \\
\hline
\end{tabular}

\section{METHODS}

\section{DNA isolation and taxonomy identification}

Genomic DNA was isolated from caudal fin-clip samples from a C. macropomum specimen obtained from the germplasm bank maintained by the National Center for Research and Conservation of Freshwater Aquatic Biodiversity of the Brazilian Ministry of the Environment. The specimen was a $3.5-\mathrm{kg}$ female (Figure 1). To confirm the taxonomic status of the specimen used in this work, we carried out (i) an external morphological evaluation [17], and (ii) a preliminary genetic analysis of an initial Illumina run for C. macropomum using the $k$-mer-matching tool Seal from the BBTools package (v 37.90, RRID:SCR_016968) [18]. We downloaded the sequences of one mitochondrial and four nuclear genes of $C$. macropomum and its two close relatives, Piaractus brachypomus and P. mesopotamicus (Table 1). Then, we used Seal to ascertain the number of reads with exclusive $k$-mers matching the sequences of each species. Out of 264,813,582 reads, 1278 matched C. macropomum, 62 matched $P$. brachypomus, and none matched $P$. mesopotamicus, confirming the identity of the samples.

\section{Sequencing and assembly}

Different data types were produced for the genome assembly of $C$. macropomum. High-molecular-weight DNA was extracted from muscle and fin clip using the MagMAX CORE nucleic acid purification kit (Thermo Fisher Scientific, Carlsbad, CA, USA) to produce PacBio continuous long reads (CLR) and Illumina paired and jumping reads (Table 2). The produced libraries were sequenced with both PacBio Single Molecule Real-Time (SMRT) sequencing technology using the Sequel system (RRID:SCR_017989) and four SMRT cells at RTL Genomics (Texas, USA), and with Illumina Hiseq2500 V4 equipment (RRID:SCR_016383) at the Functional Genomics Core Facility, Esalq-USP (São Paulo, Brazil). Illumina read quality was checked with FastQC (RRID:SCR_014583) [19] and trimmed for adaptors and low-quality bases with BBDuk (BBTools 37.90; SW15-20, RRID:SCR_016969). Genome size and heterozygosity were estimated by $k$-mer $(k=21)$ analysis (Figure $2 \mathrm{~A}$ ), performed with the sequenced Illumina data using meryl kmer counter (v1.3) [20], implemented in Canu assembler [21] and GenomeScope [22]. 
A

\section{GenomeScope Profile}

len:1,161,946,472bp uniq:78.3\% het:0.627\% kcov:19 err:0.391\% dup:2.5\% k:21

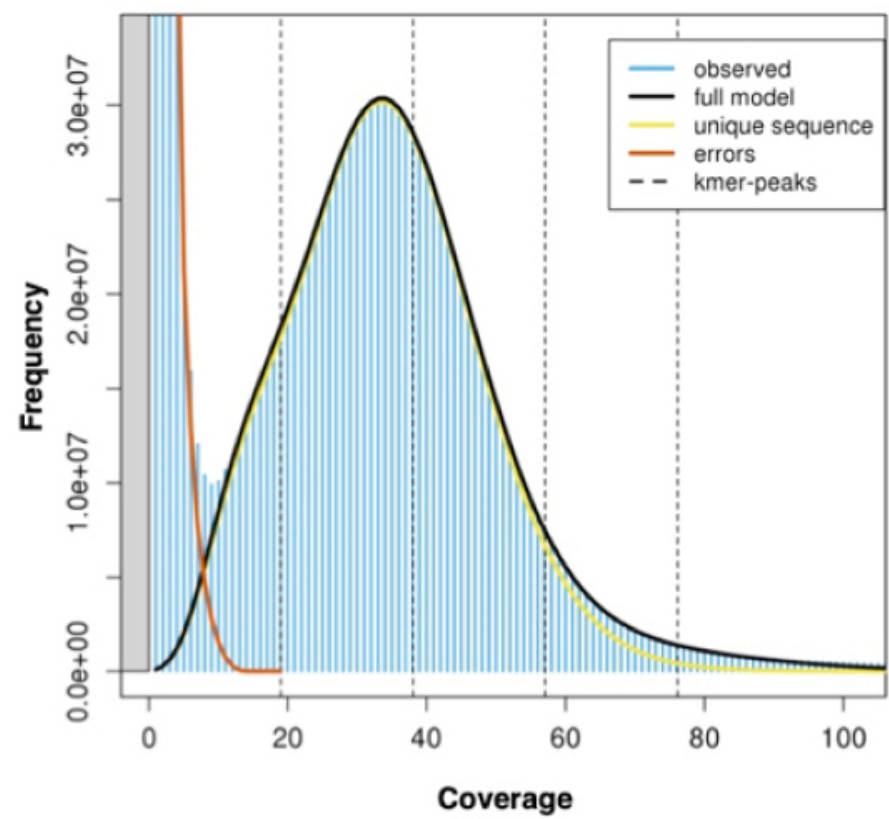

B

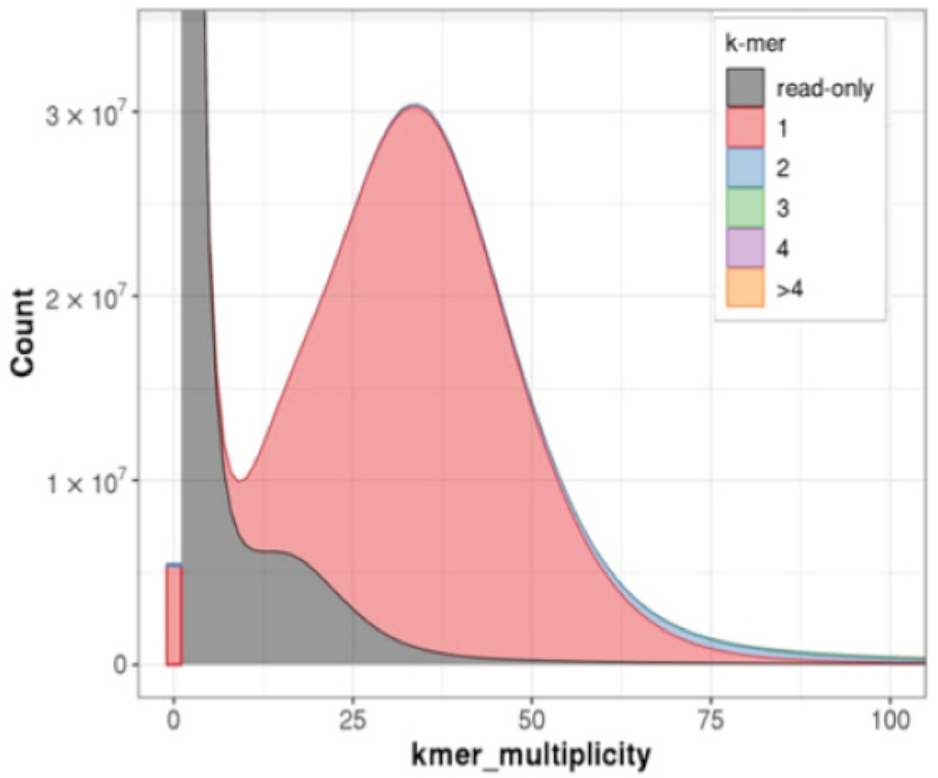

Figure 2. (A) $k$-mer composition of sequenced short Illumina reads (Table 3) of the tambaqui Colossoma macropomum. (B) A merqury $k$-mer analysis of the final tambaqui genome bases against its sequenced Illumina reads.

\begin{tabular}{|c|c|c|c|c|c|c|}
\hline Library type & Insert size (bp) & Raw data (Gbp) & Clean data (Gbp) & $\begin{array}{l}\text { Average read length } \\
\text { (bp) }\end{array}$ & N50 read length (bp) & $\begin{array}{c}\text { Clean data sequencing } \\
\text { coverage }(x)\end{array}$ \\
\hline Illumina (R1 and R2) & 400 & 59.08 & 52.93 & 100 & - & 44.89 \\
\hline Illumina (R1 and R2) & 4000 & 78.81 & 57.69 & 81 & - & 49.7 \\
\hline Illumina (R1 and R2) & 8000 & 55.59 & 41.31 & 83 & - & 35.6 \\
\hline Pacbio CLR & - & 45.58 & - & 9749 & 17667 & 39.2 \\
\hline Total & & & & & & 169.39 \\
\hline
\end{tabular}

The 21-mer distribution of the Illumina data obeyed the theoretical Poisson distribution (Figure 2A). The genome size was estimated as $1.16 \mathrm{Gbp}$ (gigabase pairs) with heterozygosity of $0.62 \%$. Based on these estimations, we sequenced a $39 \times$ coverage of the tambaqui genome in long PacBio reads, and 130× in short Illumina reads (Table 3). For the genome assembly, PacBio reads were input to the assembler Flye (v2.5, RRID:SCR_017016) [23] with the parameters "genome-size 1.5g - pacbio-raw". Then, the assembly was polished using the Illumina reads with Pilon software (RRID:SCR_014731) [24], and the parameters "frags" for paired reads and "jumps" for mate-pair reads. Finally, the assembly of the tambaqui had one round of purging with Purge_Dups (RRID:SCR_021173) [25]. Purging was performed to remove any sequences representing duplicated portions of a chromosome, which can be erroneously kept in assemblies when the divergence level of those regions in both haplotypes is high. This removed 1,167 contigs and $26 \mathrm{Mbp}$ (megabase pairs) of haplotypic 


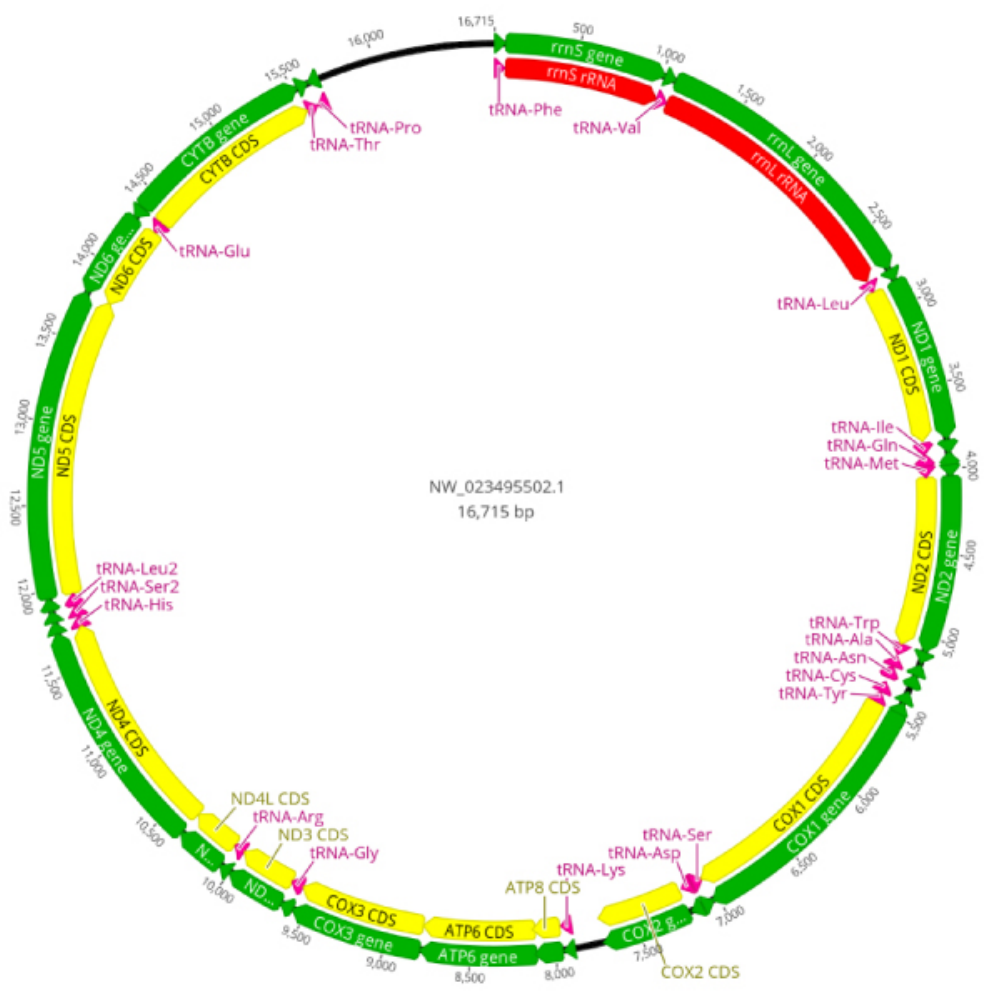

Figure 3. Mitogenome of Colossoma macropomum.

retention. The final tambaqui genome was assembled into 1,269 scaffolds with a scaffold N50 of $40 \mathrm{Mbp}$ and a total assembly length of 1,221,847,006 bp (Table 2). A fraction of 93\% of the genome is assembled on 54 scaffolds, which represent the main tambaqui karyotype [26]. We have also identified the mitochondrial genome (Figure 3) within our assembled genome: it is represented by scaffold NW_023495502.1, which is 16,715 bp in length and has conserved gene content and synteny with the $C$. macropomum mitogenome available at the National Center for Biotechnology Information (NCBI; KP188830.1).

\section{Repeat sequences and gene annotation}

We identified repeat sequences in C. macropomum using homology-based, and de novo approaches. A de novo library of repeats was created for the tambaqui using RepeatModeler2 package (RRID:SCR_015027) [27]. This library was then combined with RepBase (release 26.04, RRID:SCR_021169) [28], forming the final "teleost” library with which $C$. macropomum genome repeats were searched. Table 4 presents the repeat summary of $C$. macropomum: $52.49 \%$ of the genome comprises repeats, of which $49.78 \%$ are interspersed repeats. The $C$. macropomum genome was submitted to NCBI for annotation. The robust NCBI Eukaryotic Annotation Pipeline uses homology-based and ab initio gene prediction to annotate genes (including protein-coding and non-coding, such as lncRNAs and snRNAs), pseudogenes, transcripts, and proteins. Details of the pipeline are described in the NCBI Annotation HandBook [29]. Briefly: first, repeats are masked with RepeatMasker (RRID:SCR_012954) [30] and Window Masker [31]. Subsequently, transcripts, proteins, and RNA-seq data from the NCBI database are aligned to the genome with 


\begin{tabular}{|c|c|c|c|c|}
\hline $\begin{array}{l}\text { Bases masked: } 641,307,197 \\
\text { bp }(52.49 \%)\end{array}$ & & $\begin{array}{l}\text { Number of } \\
\text { elements* }\end{array}$ & Length occupied & $\%$ of sequence \\
\hline \multirow[t]{15}{*}{ Retroelements } & & 131365 & 35210915 & 2.88 \\
\hline & SINEs: & 3369 & 162823 & 0.01 \\
\hline & Penelope & 2614 & 206056 & 0.02 \\
\hline & LINEs: & 88299 & 25531727 & 2.09 \\
\hline & CRE/SLACS & 5 & 195 & 0 \\
\hline & L2/CR1/Rex & 54941 & 16069764 & 1.32 \\
\hline & R1/LOA/Jockey & 1613 & 158940 & 0.01 \\
\hline & $\mathrm{R} 2 / \mathrm{R} 4 / \mathrm{NeSL}$ & 688 & 137427 & 0.01 \\
\hline & RTE/Bov-B & 9260 & 3512602 & 0.29 \\
\hline & L1/CIN4 & 9819 & 2801917 & 0.23 \\
\hline & LTR elements: & 39697 & 9516365 & 0.78 \\
\hline & $\mathrm{BEL} / \mathrm{Pao}$ & 1824 & 655410 & 0.05 \\
\hline & Ty1/Copia & 3452 & 196980 & 0.02 \\
\hline & Gypsy/DIRS1 & 17593 & 6224074 & 0.51 \\
\hline & Retroviral & 13302 & 1948492 & 0.16 \\
\hline \multirow[t]{6}{*}{ DNA transposons } & & 428117 & 94637950 & 7.75 \\
\hline & hobo-Activator & 50751 & 5464720 & 0.45 \\
\hline & Tc1-IS630-Pogo & 270090 & 78887086 & 6.46 \\
\hline & PiggyBac & 3206 & 517597 & 0.04 \\
\hline & Tourist/Harbinger & 4980 & 440554 & 0.04 \\
\hline & $\begin{array}{l}\text { Other (Mirage, } \\
\text { P-element, Transib) }\end{array}$ & 1414 & 117503 & 0.01 \\
\hline Rolling circles & & 9904 & 2012553 & 0.16 \\
\hline Unclassified: & & 2468233 & 478402494 & 39.15 \\
\hline Total interspersed repeats & & & 608251359 & 49.78 \\
\hline Small RNA: & & 2641 & 167105 & 0.01 \\
\hline Satellites: & & 15326 & 2676106 & 0.22 \\
\hline Simple repeats: & & 435230 & 23721925 & 1.94 \\
\hline Low complexity & & 51965 & 4532860 & 0.37 \\
\hline
\end{tabular}

Splign [32] and ProSplign [33]. Those alignments are submitted to Gnomon [34] for gene prediction. Gnomon (i) merges non-conflicting alignments into putative models, then (ii) extends predictions missing a start and a stop codon or internal exon(s) using a hidden Markov model (HMM) algorithm. Finally, Gnomon (ii) builds pure ab initio predictions where it finds open reading frames of sufficient length but with no supporting alignment detected. Models built on RefSeq transcript alignments are given preference over overlapping Gnomon models with the same splice pattern. Table 5 presents a summary of the annotation of $C$. macropomum. A detailed description of the tambaqui genome annotation can be found on the NCBI Eukaryotic Annotation Page [35].

\section{RESULTS AND DISCUSSION}

All sequencing data are available at the NCBI under the BioProject PRJNA702552, via Sequence Read Archive (SRA) accession numbers SRX10122091 to SRX10122101. The assembled genome and sequence annotations are available at the NCBI with the accession number GCF_904425465.1. The genome sequence and the annotation files-including CDS and proteins_-can be downloaded from the NCBI FTP server [37]. Finally, a genome DataViewer was built for the tambaqui [38]. This browser is ideal for 


\begin{tabular}{lc}
\hline Table 5. Summary of the annotated features of Colossoma macromapum genome. \\
\hline Feature & Colossoma macropomum \\
\hline Genes and pseudogenes & 31,149 \\
Protein-coding & 26,670 \\
Non-coding & 3279 \\
CDSs & 43,938 \\
Fully-supported & 1648 \\
With $>$ 5\% ab initio & 267 \\
Partial & 43,618 \\
Protein assigned RefSeq(XP) & 2011 \\
Mean CDS length (bp) & 2631 \\
Mean intron length (bp) & 280 \\
Mean exon length (bp) & 12.02 \\
\hline Mean exon per gene & \\
\hline Detailed annotation report can be found at [36]. & \\
\hline
\end{tabular}

further exploration of the tambaqui genome, especially by those who are not specialist bioinformaticians, such as geneticists working on selective breeding programs.

\section{Evaluating the completeness of the genome assembly and annotation}

The final assembly of the tambaqui is $1.2 \mathrm{Gbp}$ with a scaffold N50 size of $40.163 \mathrm{Mbp}$ (Table 2). Figure 2A shows the DNA $k$-mer prediction of genome size done using the Illumina reads produced to polish this assembly. Further, Figure 2B presents a merqury [39] $k$-mer plot of the final assembly: merqury produces a mapping-free evaluation of $k$-mer completeness in genomes by comparing the assembly $k$-mers with raw reads for the specimen. In this case, we used the high-quality Illumina reads (Table 3) to plot the merqury evaluation against the genome $k$-mers. Figure 2B shows that (i) the $k$-mers in the genome are in accordance with its Illumina read $k$-mers, (ii) the assembly $k$-mers have the same distribution of the raw reads $k$-mer (2A), and that (iii) most of the assembly $k$-mers (pink color) are unique in the genome, showing that the final assembly of the tambaqui has low levels of haplotypic retention (blue color). The final phred-like merqury QV score is $36.73(\mathrm{QV}=36.73)$, meaning that the tambaqui assembled bases are more than $99.9 \%$ accurate. The merqury completeness score shows that $89.31 \%$ of kmers in the Illumina reads are present in the assembly, which is a good recovery of $k$-mers for a species with $0.6 \%$ heterozygosity.

For the tambaqui genome, 93\% of the assembled bases are present in the largest 54 scaffolds. We performed a first nucleotide synteny analysis of Benchmarking Universal Single-Copy Ortholog (BUSCO) genes found in the first 54 scaffolds of $C$. macropomum against the BUSCO genes on genome of Ictalurus punctatus [40] using busco2fasta [41] and Circos [42]. The synteny is presented in Figure 4. C. macropomum and I. punctatus shared a common ancestor 150 million years ago [43]. The image shows a good degree of synteny in terms of BUSCO genes; for a number of times entire chromosomes are syntenic. Figures 5 and 6 show similar analysis with C. auratus [44] and Astyanax mexicanus [45] of different levels of relatedness to $C$. macropomum, demonstrating the potential of this highly contiguous genome for studies of chromosome evolution.

Finally, we performed a general gene presence analysis of the $C$. macropomum genome using BUSCO software (v5.0.0, RRID:SCR_015008) [46] and the OrthoDB 


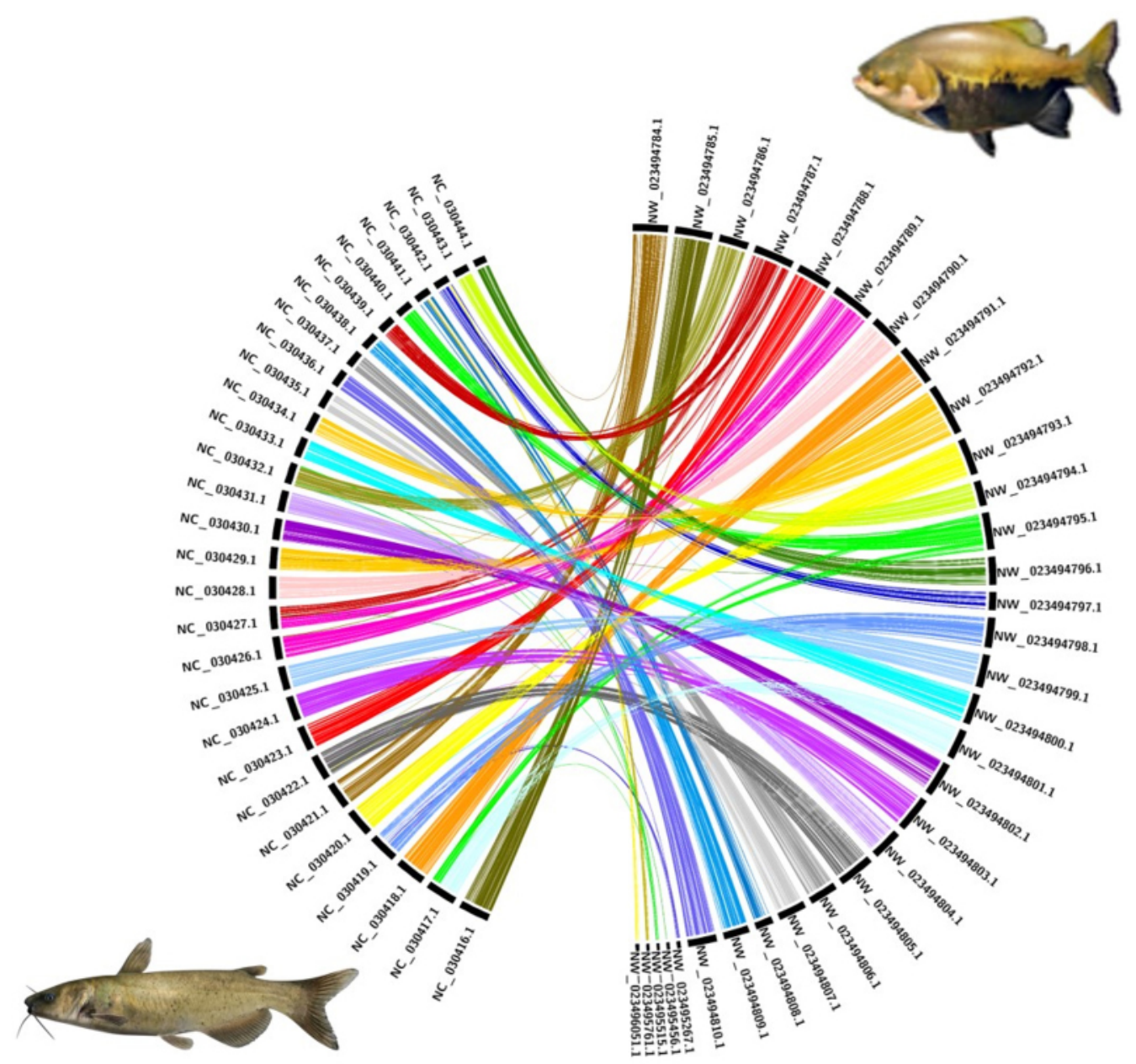

Figure 4. BUSCO gene synteny of Colossoma macropomum (tambaqui; right) and Ictalurus punctatus (channel catfish; left). Synteny analysis of single copy genes reveals low conservation of homologous gene order between the species. Most $C$. macropomum genes are pulverized into several linkage groups of the I. punctatus genome, which may reflect the different genome evolutionary events experienced by these species.

(RRID:SCR_011980) [47] library actinopterygii_odb10. BUSCO v5 recovered 96.5\% of complete BUSCO genes out of 3640 genes searched, where $95.5 \%$ were complete and single copy, $1.0 \%$ were duplicated, $1.0 \%$ were fragmented, and $2.5 \%$ were missing. Once again, this demonstrates the quality of the tambaqui assembly.

\section{Gene family identification and phylogenetic analysis of}

\section{C. macropomum}

To identify gene families among $C$. macropomum and other species, we downloaded the whole genome predicted protein sequences from the NCBI Eukaryotic Annotation Page of Oreochromis niloticus (GCF_001858045.2), Carassius auratus (GCF_003368295.1), Danio rerio (GCF_000002035.6), Latescalcarifer (GCF_001640805.1), Cyprinus carpio (GCF_000951615.1), Rhincodontypus (GCF_001642345.1), Poecilia formosa (GCF_000485575.1), Ictalurus punctatus (GCF_001660625.1), Astyanax mexicanus (GCF_000372685.2), 


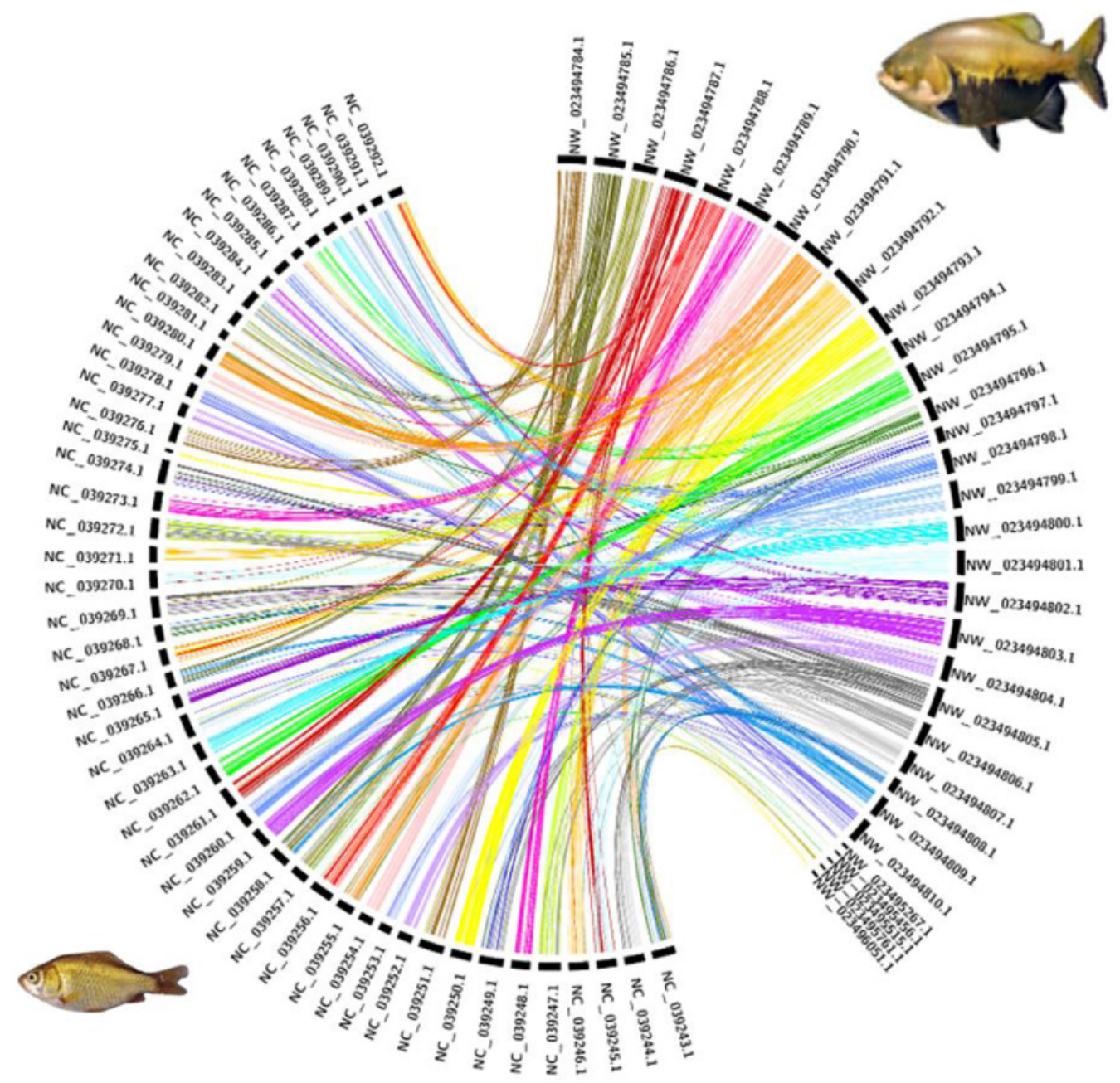

Figure 5. BUSCO gene synteny of Colossoma macropomum (tambaqui; right) and Carassius auratus (goldfish; left). Synteny analysis of single copy genes reveals low conservation of homologous gene order between the species. Most $C$. macropomum genes are pulverized into several linkage groups of the $C$. auratus genome, reflecting the different genome evolutionary events experienced by these species.

Oncorhynchus mykiss (GCF_013265735.2) and Pygocentrus nattereri (GCF_001682695.1). We then input this data to Orthofinder (v2.5.2) [48]. Of all the proteins imputed from the 12 species, Orthofinder assigned $97.3 \%$ of the proteins to 31,794 orthogroups. There were 10,939 orthogroups with all the species present, and 33 of them consisted of single-copy genes. Those 33 single-copy orthologs were used to generate a phylogeny (Figure 7). First, single-copy genes were aligned with MAFFT (v7.455, RRID:SCR_011811) [49], and alignments were trimmed with trimAL (v1.4. rev15, RRID:SCR_017334) [50]. Then, a supermatrix was created using geneStitcher.py [51], which was imputed to PhyML (RRID:SCR_014629) [52] for a phylogeny with the amino acid substitution model LG and 100 bootstrap replicates. The phylogeny presented herein (Figure 7) is consistent with other studies [53, 54]. 


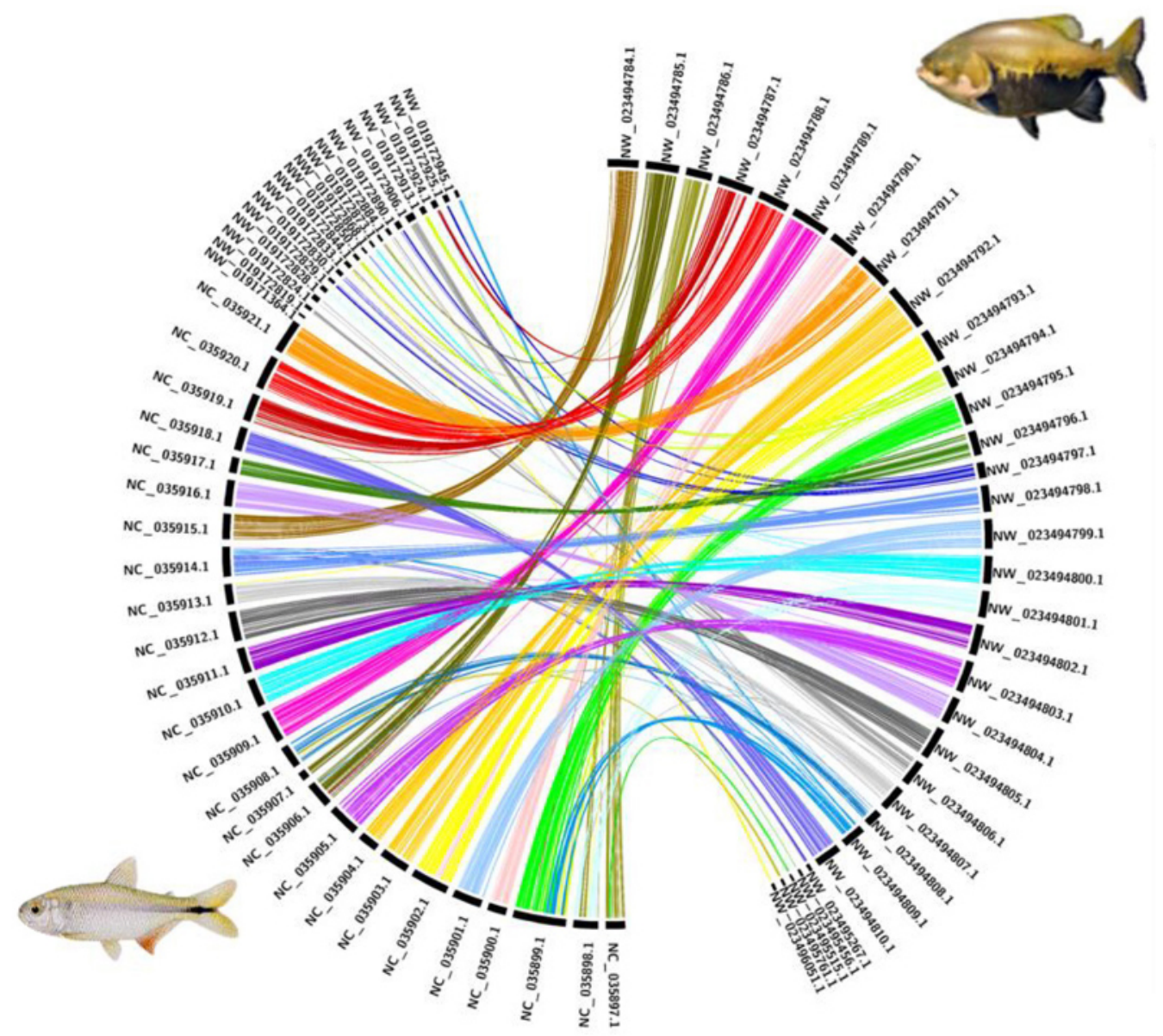

Figure 6. BUSCO gene synteny of Colossoma macropomum (tambaqui; right) and Astyanax mexicanus (Mexican tetra; left). Synteny analysis confirms moderate conservation of homologous single copy gene order between these species. While large syntenic blocks persist, a relatively large portion of $C$. macropomum genes are also fragmented into two or more linkage groups of the A. mexicanus genome.

\section{Re-use Potential}

Seasonal and long-term modifications in environmental conditions are well-known to be associated with periodic events of low water dissolved oxygen leading to hypoxia and even anoxia. Tambaqui is an Amazon fish species that has developed adaptions to deal with this, such as enlargement of the lower lip to grasp oxygen better to survive in hypoxic conditions. These, along with other fish adaptations to the Amazon aquatic ecosystem, are intriguing scientific questions that could be scientifically addressed using the present well-assembled and annotated tambaqui genome. Moreover, the availability of this annotated genome will pave the way for the development of tools for genomic breeding programs of tambaqui, the most important native species for aquaculture in South America.

\section{DATA AVAILABILITY}

The data sets supporting the results of this article are available in the GigaScience Database [55]. 
a

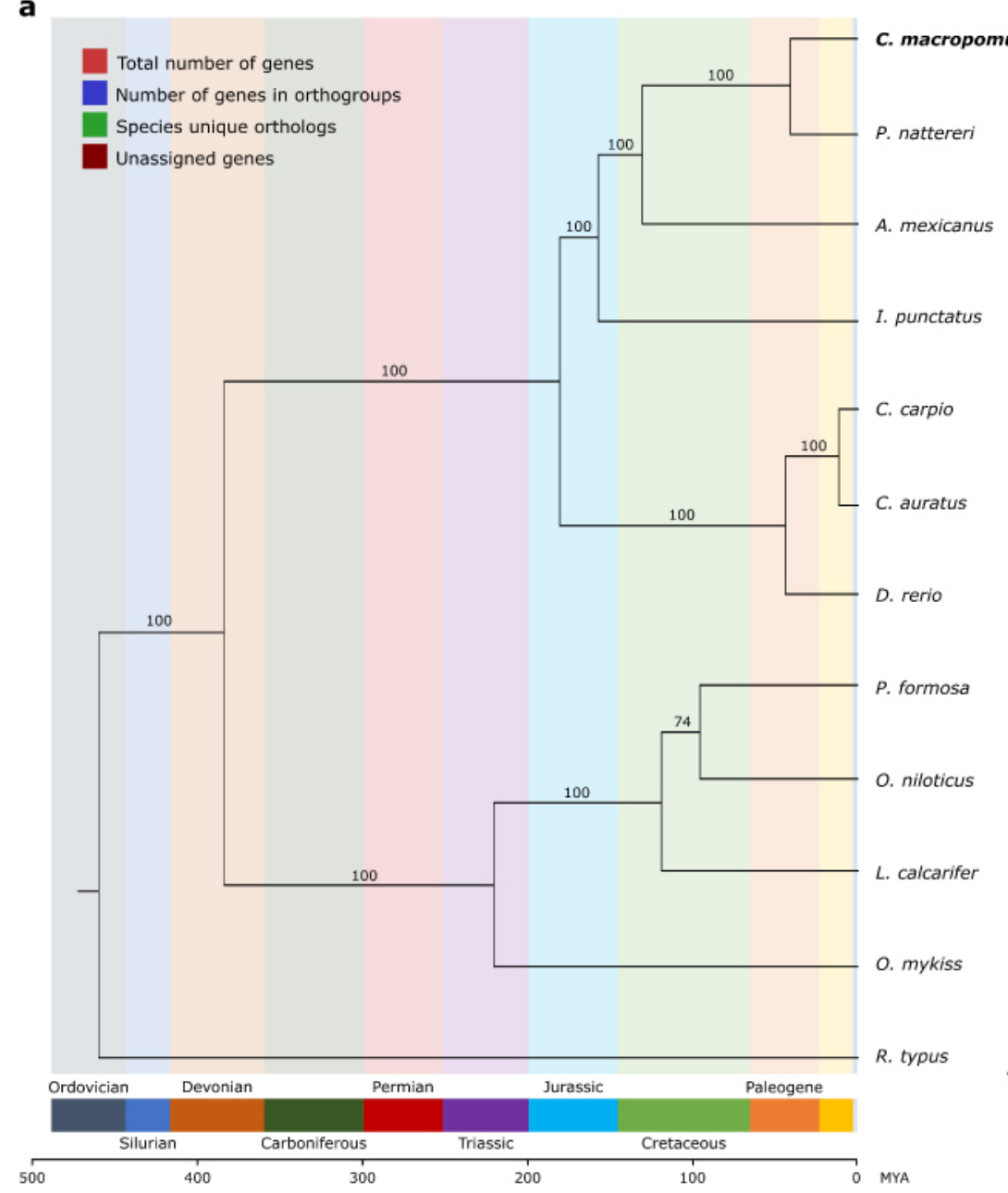

(2)
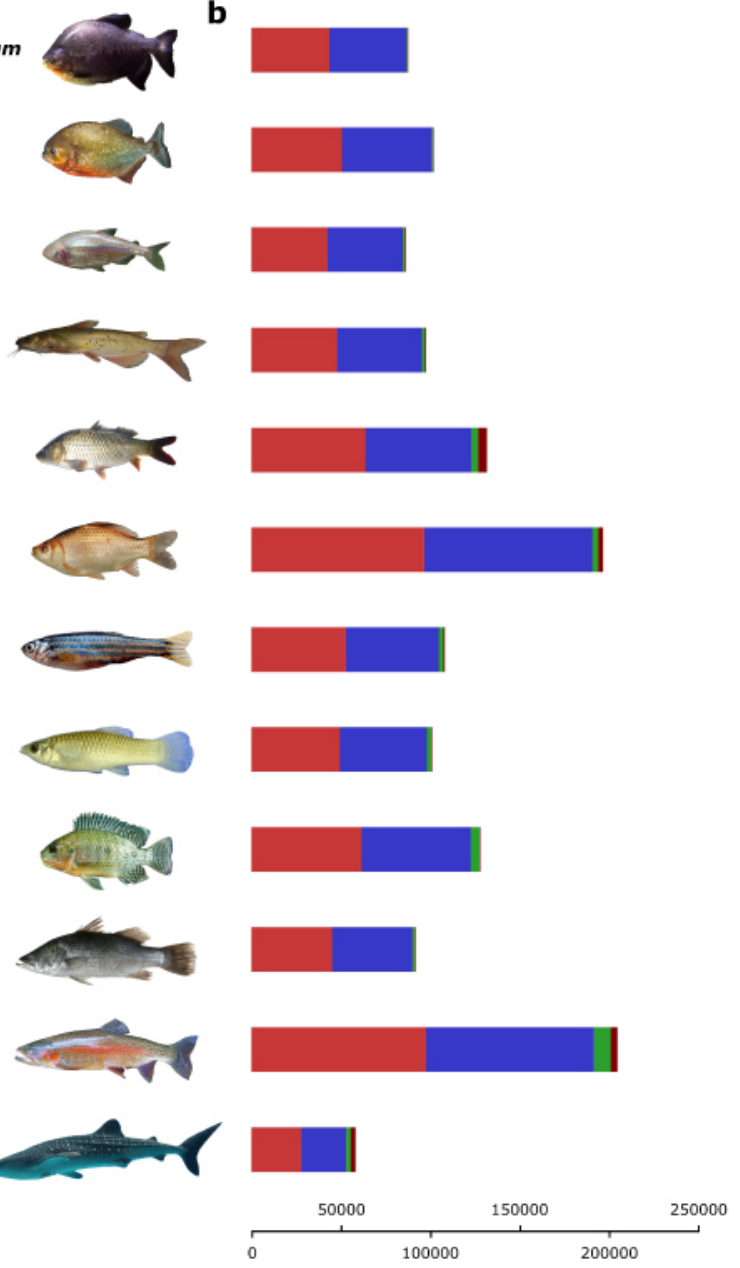

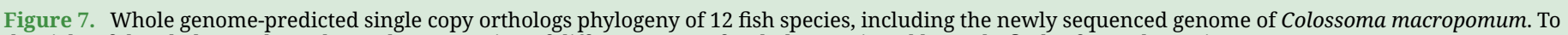
the right of the phylogeny bars shows the proportion of different types of orthologs assigned by Orthofinder for each species.

All sequencing data is available on NCBI under the BioProjects PRJNA702552 and PRJEB40318. The former contains the Sequence Read Archive (SRA) experiments with accession numbers SRX10122091 to SRX10122101. The latter comprises the assembled genome and sequence annotations with the accession number GCF_904425465.1.

The genome sequence and annotation files-including coding sequences and proteins-can be downloaded from the NCBI FTP server [37]. A data viewer is also available [38].

\section{DECLARATIONS}

\section{LIST OF ABBREVIATIONS}

BUSCO: Benchmarking Universal Single-Copy Ortholog; Gbp: gigabase pair(s); Mbp: megabase pair(s); QV: consensus quality value. 


\section{ETHICAL APPROVAL}

We followed the applicable international and national ethical guidelines for the care and use of animals in research. The approval of the Ethics Committee for the Use of Animal registration is placed at the University of Mogi das Cruzes and is numbered \#019/2017.

\section{COMPETING INTERESTS}

The authors declare that they have no competing interests.

\section{FUNDING}

The authors acknowledge FAPESP (São Paulo Research Foundation \#2015/23883-0), National Council for Scientific and Technological Development (CNPq), and Coordination of Superior Level Staff Improvement (CAPES) through Tambaqui Project (Edital Pró-Amazonia 047/2012) for financial support. AWSH, LLC, VWDAV, and DP are recipients of CNPq productivity scholarships (304662/2017-8, 304353/2019-1, 306718/2019-7, and 312273/2017-7, respectively).

\section{AUTHORS" CONTRIBUTIONS}

AWSH, LLC, and DP designed and conceived this work; AWSH collected the samples; AWSH, MUS, DP, LLC, VMDAV wrote the manuscript; MUS and HM coordinated and carries out the bioinformatics analyses; AWSH, LLC and DP revised the manuscript. All authors read and approved the final manuscript.

\section{ACKNOWLEDGEMENTS}

We acknowledge CEPTA-ICMBio (Centro Nacional de Pesquisa e Conservação da Biodiversidade Aquática Continental) for tambaqui individual contributions to this work, and Dr. Andrew Veale for critical review and language editing.

\section{REFERENCES}

1 Jézéquel C et al. A database of freshwater fish species of the Amazon Basin. Sci. Data, 2020; 7: 96.

2 Goulding M, Carvalho ML, Life history and management of the tambaqui (Colossoma macropomum, Characidae): an important Amazonian food fish. Rev. Bras. Zool., 1982; 1: 107-133.

3 Anderson JT, Nuttle T, Saldaña-Rojas JS, Pendergast TH, Flecker AS, Extremely long-distance seed dispersal by an overfished Amazonian frugivore. Proc. Biol. Sci., 2011; 278: 3329-3335.

4 Araújo-Lima CARM, Ruffino ML, Peixes migradores da Amazônia brasileira. In: Carolsfield J, Harvey B, Ross C, Baer A (eds), Peixes migradores da América do Sul. Biologia, Pesca e Estado de Conservação. World Fisheries Trust, International Development Research Centre and Banco Mundial, 2003; pp. 233-302.

5 Sousa RGC, Freitas CEC, Seasonal catch distribution of tambaqui (Colossoma macropomum), Characidae in a central Amazon floodplain lake: implications for sustainable fisheries management. $J$. Appl. Ichthyol., 2011; 27: 118-121.

6 Instituto Brasileiro de Geografia e Estatística (IBGE). Aquicultura. In: Produção Pecuária Municipal. Rio de Janeiro: IBGE, 2020; https://sidra.ibge.gov.br/tabela/3940. Accessed 09 Nov 2020.

7 Prado-Lima M, Val AL, Transcriptomic characterization of tambaqui (Colossoma macropomum, Cuvier, 1818) exposed to three climate change scenarios. PLoS One, 2016; 11: e0152366.

8 Fé-Gonçalves LM et al. Transcriptomic evidences of local thermal adaptation for the native fish Colossoma macropomum (Cuvier, 1818). Genet. Mol. Biol., 2020; 43: e20190377.

9 Lobo IKC et al. Transcriptome of tambaqui Colossoma macropomum during gonad differentiation: Different molecular signals leading to sex identity. Genomics, 2020; 112: 2478-2488. 
10 Ferraz RB et al. A complete enzymatic capacity for long-chain polyunsaturated fatty acid biosynthesis is present in the Amazonian teleost tambaqui, Colossoma macropomum. Comp. Biochem. Physiol. B Biochem. Mol. Biol., 2019; 227: 90-97.

11 Ferraz RB et al. The fatty acid elongation genes elovl $4 a$ and elovl $4 \mathrm{~b}$ are present and functional in the genome of tambaqui (Colossoma macropomum). Comp. Biochem. Physiol. B Biochem. Mol. Biol., 2020; 245: 110447.

12 Nunes JRS et al. Large-scale SNP discovery and construction of a high-density genetic map of Colossoma macropomum through genotyping-by-sequencing. Sci. Rep., 2017; 7: 46112.

13 Gomes $\mathbf{F}$ et al. Identification and characterization of the expression profile of the microRNAs in the Amazon species Colossoma macropomum by next generation sequencing. Genomics, 2017; 109: 67-74.

14 Perazza CA, Menezes JTB, Ferraz JBS, Pinaffi FLV, Silva LA, Hilsdorf AWS, Lack of intermuscular bones in specimens of Colossoma macropomum: an unusual phenotype to be incorporated into genetic improvement programs. Aquaculture, 2017; 472(Suppl 1): 57-60.

15 Nunes JRS et al. Genome-wide association study reveals genes associated with the absence of intermuscular bones in tambaqui (Colossoma macropomum). Anim. Genet., 2020; 51: 899-909.

16 Hilsdorf AWS, Hallerman E, Genetic resources of neotropical fishes. 1st ed., Heidelberg: Springer, 2017. ISBN 978-3-319-55836-3.

17 Géry J, Characoids of the world. Neptune City, NJ: TFH Publications, 1977.

18 Bushnell B, BBTools: A suite of fast, multithreaded bioinformatics tools designed for analysis of DNA and RNA sequence data. 2018; https://jgi.doe.gov/data-and-tools/bbtools/.

19 Andrews S, FastQC: a quality control tool for high throughput sequence data. 2010; http://www.bioinformatics.babraham.ac.uk/projects/fastqc.

20 Maryland Bioinformatics Labs. meryl (version 1.3). 2021; https://github.com/marbl/meryl.

21 Koren S, Walenz BP, Berlin K, Miller JR, Bergman NH, Phillippy AM, Canu: scalable and accurate long-read assembly via adaptive $k$-mer weighting and repeat separation. Genome Res., 2017; 27: $722-736$.

22 Vurture GW et al. GenomeScope: fast reference-free genome profiling from short reads. Bioinformatics, 2017; 33: 2202-2204.

23 Kolmogorov M, Yuan J, Lin Y, Pevzner PA, Assembly of long, error-prone reads using repeat graphs. Nat. Biotechnol., 2019; 37: 540-546.

24 Walker BJ et al. Pilon: an integrated tool for comprehensive microbial variant detection and genome assembly improvement. PLoS One, 2014; 9: e112963.

25 Guan D, McCarthy SA, Wood J, Howe K, Wang Y, Durbin R, Identifying and removing haplotypic duplication in primary genome assemblies. Bioinformatics, 2020; 36: 2896-2898.

26 Nakayama CM, Feldberg E, Bertollo LAC, Karyotype differentiation and cytotaxonomic considerations in species of Serrasalmidae (Characiformes) from the Amazon basin. Neotrop. Ichthyol., 2012; 10: 53-58.

27 Flynn JM et al. RepeatModeler2 for automated genomic discovery of transposable element families. Proc. Natl Acad. Sci. USA, 2020; 117: 9451-9457.

28 Bao W, Kojima KK, Kohany O, Repbase Update, a database of repetitive elements in eukaryotic genomes. Mob. DNA, 2015; 6: 11.

29 National Center for Biotechnology Information (NCBI). Eukaryotic genome annotation guide. Bethesda, MD: NCBI;

https://www.ncbi.nlm.nih.gov/genbank/eukaryotic_genome_submission_annotation/.

30 Smit A, Hubley R, Green P, RepeatMasker Open-4.0. 2013-2015. 2015; http://www.repeatmasker.org.

31 Morgulis A, Gertz EM, Schäffe AA, Agarwala R, WindowMasker: window-based masker for sequenced genomes. Bioinformatics, 2006; 22: 134-141.

32 Kapustin Y, Souvorov A, Tatusova T, Lipman D, Splign: algorithms for computing spliced alignments with identification of paralogs. Biol. Direct., 2008; 3: 20.

33 National Center for Biotechnology Information (NCBI). ProSplign. Bethesda, MD: NCBI. 2017; https://www.ncbi.nlm.nih.gov/sutils/static/prosplign/prosplign.html. 
34 Souvorov A, Kapustin Y, Kiryutin V, Chetvernin V, Tatusova T, Lipman D, Gnomon-NCBI eukaryotic gene prediction tool. Bethesda, MD: NCBI. 2010;

https://www.ncbi.nlm.nih.gov/core/assets/genome/files/Gnomon-description.pdf.

35 National Center for Biotechnology Information (NCBI). NCBI Colossoma macropomum annotation release 100. Bethesda, MD: NCBI. 2020; https://www.ncbi.nlm.nih.gov/genome/annotation_euk/Colossoma_macropomum/100/.

36 National Center for Biotechnology Information (NCBI). NCBI Colossoma macropomum annotation release 100. Gene and feature statistics. Bethesda, MD: NCBI. 2020; https://www.ncbi.nlm.nih.gov/genome/annotation_euk/Colossoma_macropomum/100/\#BuildInfo.

37 National Center for Biotechnology Information (NCBI). Index of /genomes/all/GCF/904/425/465/GCF_904425465.1_Colossoma_macropomum. Bethesda, MD: NCBI. 2020; https:/ftp.ncbi.nlm.nih.gov/genomes/all/GCF/904/425/465/GCF_904425465.1_Colossoma_macropomum/.

38 National Center for Biotechnology Information (NCBI). Genome data viewer. Colossoma macroponum (tambaqui). Bethesda, MD: NCBI. https://www.ncbi.nlm.nih.gov/genome/gdv/browser/genome/?id=GCF_904425465.1.

39 Rhie A, Walenz BP, Koren S, Philippy AM, Merqury: reference-free quality, completeness, and phasing assessment for genome assemblies. Genome Biol., 2020; 21: 245.

$40 \mathrm{Liu} \mathrm{Z}$ et al. The channel catfish genome sequence provides insights into the evolution of scale formation in teleosts. Nat. Commun., 2016; 7: 11757.

41 Stevens L, busco2fasta. 2021; https://github.com/lstevens17/busco2fasta.

42 Krzywinski MI et al. Circos: an information aesthetic for comparative genomics. Genome Res., 2009; 19: 1639-1645.

43 Betancur RR et al. Phylogenetic classification of bony fishes. BMC Evol. Biol., 2017; 17: 162.

44 Chen $\mathrm{Z}$ et al. De novo assembly of the goldfish (Carassius auratus) genome and the evolution of genes after whole-genome duplication. Sci. Adv., 2019; 5: eaav0547.

45 Warren WC et al. A chromosome-level genome of Astyanax mexicanus surface fish for comparing population-specific genetic differences contributing to trait evolution. Nat. Commun., 2021; 12: 1447.

46 Waterhouse RM et al. BUSCO applications from quality assessments to gene prediction and phylogenomics. Mol. Biol. Evol., 2018; 35: 543-548.

47 Zdobnov EM et al. OrthoDB v9.1: cataloging evolutionary and functional annotations for animal, fungal, plant, archaeal, bacterial and viral orthologs. Nucleic Acids Res., 2017; 45: D744-D749.

48 Emms DM, Kelly S, OrthoFinder: phylogenetic orthology inference for comparative genomics. Genome Biol., 2019; 20: 238 .

49 Katoh K, Standley DM, MAFFT multiple sequence alignment software version improvements in performance and usability. Mol. Biol. Evol., 2013; 30: 772-780.

50 Capella-Gutiérrez S, Silla-Martínez JM, Gabaldón T, trimAl: a tool for automated alignment trimming in large-scale phylogenetic analyses. Bioinformatics, 2009; 25: 1972-1973.

51 Ballesteros JA, Hormiga GA, New orthology assessment method for phylogenomic data: unrooted phylogenetic orthology. Mol. Biol. Evol., 2016; 33: 2117-2134.

52 Guindon S, Dufayard J-F, Lefort V, Anisimova M, Hordijk W, Gascuel O, New algorithms and methods to estimate maximum-likelihood phylogenies: assessing the performance of PhyML 3.0. Syst. Biol., 2010; 59: 307-321.

53 Steinke D, Salzburger W, Meyer A, Novel relationships among ten fish model species revealed based on a phylogenomic analysis using ESTs. J. Mol. Evol., 2006; 62: 772-784.

54 Hughes LC et al. Comprehensive phylogeny of ray-finned fishes (Actinopterygii) based on transcriptomic and genomic data.Proc. Natl Acad. Sci. USA, 2018; 115: 6249-6254.

55 Hilsdorf AWS, Uliano-Silva M, Coutinho LL, Montenegro H, Almeida-Val VMF, Pinhal D, Supporting data for "Genome assembly and annotation of the tambaqui (Colossoma macropomum): an emblematic fish of the Amazon River basin”. GigaScience Database. 2021; http://dx.doi.org/10.5524/100933. 